\title{
RESULTADOS ACADÉMICOS OBTENIDOS A TRAVÉS DE PROPUESTAS PEDAGÓGICAS INNOVADORAS PARA LA PRODUCCIÓN DE LAS TESINAS
}

MÓNICA I. CESANA BERNASCONI MÓNICA A. CANTEROS

FACULTAD DE CIENCIAS ECONÓMICAS - UNNE 
RESUMEN

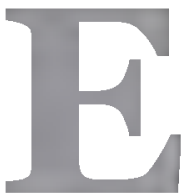

n la presente publicación nos proponemos exponer el análisis de los resultados obtenidos en relación con el proceso de producción y presentación de las tesinas de las licenciaturas, en las carreras de Economía y Administración, de la Facultad de Ciencias Económicas de la Universidad Nacional del Nordeste, frente a una problemática planteada desde la realidad académica ya que hacia el año 2006 existía una baja tasa de finalización de las tesinas y ello significaba un retraso para la graduación de dichos profesionales. Ante esta situación, buscamos diferentes propuestas que nos permitieran superar los obstáculos detectados. Aquí resumimos dicho trabajo mediante un análisis de tipo comparativo para observar los datos que hemos acumulado desde el año 2007 hasta la fecha y el efecto que hemos logrado sobre la base de distintos supuestos que guiaron nuestras propuestas de cambio, entre otros, modificaciones sobre el sistema de cursado de la materia Metodología de la Investigación, seminario con Tesina Final, con respecto al periodo anterior (2003- 2005). Es así como, sorteando un periodo de transición que tuvo lugar durante el año 2006, a partir del año 2007 nos propusimos implementar, como primera estrategia superadora, el diseño del Plan de Tesina durante el cursado de la materia y, concomitante, como requisito de regularidad de ésta. El diseño se llevó a cabo con el objetivo de superar una etapa signada por el decaimiento de los niveles de producción científica de los trabajos de tesis de la Facultad. Asimismo, a partir del año 2007, implementamos el sistema de tutorías virtuales, las que fueron evolucionando, hasta que en 2009 se combinaron la modalidad presencial y la virtual. La primera de ellas fue la que nos permitió realizar un trabajo de campo sobre esta estrategia de enseñanza-aprendizaje, que en cada año fue mejorándose desde la práctica. Las mejoras fueron el resultado del feed-back con los alumnos. Las tutorías presenciales nos permitieron trabajar con grupos de alumnos más numerosos y convertir esta situación en una oportunidad para el intercambio de ideas y la complementariedad de los puntos de vistas entre pares.

De este modo, dentro del periodo 2007-2011, hemos constatado empíricamente, y tanto mediante datos cuantitativos como cualitativos, el incremento del número de tesinas defendidas y, por lo tanto, igual impacto sobre la tasa de graduados de ambas licenciaturas. Por último, aunque no menos importante, hemos podido revalorizar la calidad del trabajo científico que realizan mediante la divulgación de sus trabajos, ya que en el año 2010 organizamos la "Jornada de vinculación de tesistas con el medio", e inclusive, un graduado realizó la publicación internacional de su tesina.

Como conclusión, entendemos que quedaría suficientemente demostrada la viabilidad de continuar otorgando una formación de excelencia para nuestros graduados de licenciatura, mediante un plan de estudios que incluya la formación metodológica como apoyo de lo disciplinar y un trabajo final de tesis que permita culminar la formación crítico-reflexiva del alumno, cuya opinión fundamentada enaltece a toda academia, como también a sus egresados. 


\section{METODOLOGÍA}

Nuestro trabajo posee un enfoque cuali-cuantitativo, de tipo descriptivo y comparativo, de corte longitudinal.

Como objetivo, nos proponemos exponer y evaluar el proceso de ajuste que, desde el año 2007 a la fecha, hemos realizado sobre algunas variables que intervienen en la construcción y presentación de una tesina de licenciatura, en particular, de los alumnos de la materia Metodología de la Investigación de la Facultad de Ciencias Económicas de la Universidad Nacional del Nordeste.

Para ello, recortamos dos etapas de un periodo comprendido entre los años 2003 y 2011 (a la actualidad), diferenciados por importantes cambios que van desde lo curricular, pedagógico y de estrategias didácticas, como la nueva reglamentación que rige a la cátedra. Finalmente, se observaron los valores obtenidos en cada etapa y se los interpretó como el impacto producido por cada variación en el valor de las variables cuantitativas. En el análisis, hemos podido evaluar y exponer los futuros ajustes a realizar para continuar mejorando la calidad del trabajo de la cátedra en su conjunto y de la propuesta académica de la Facultad.

\section{LA CÁTEDRA Y LA SITUACIÓN PROBLEMÁTICA A RESOLVER}

La materia Metodología de la Investigación - Seminario con tesina, hasta la actualidad, se imparte en el quinto año de las licenciaturas, en las carreras en Economía y Administración, de la Facultad de Ciencias Económicas de la Universidad Nacional del Nordeste. Esto significa que el alumno cierra su ciclo universitario con una producción científica "tesina".

En sus primeros años la materia constituía un espacio de formación cuyo objetivo principal, según exponía el Anexo I de la Resolución CD 5233/02 (15/08/02), era que los cursantes:

"Adquieran un conocimiento, un saber y un saber hacer sobre los rasgos más salientes de la práctica científica como conocimiento operatorio de modo de aprovechar la potencia del método científico para las actividades propias de sus actividades profesionales.

Adopten para sí los fines trascendentes de la producción de nuevos conocimientos, encarnando de esa manera el perfil de un profesional motivado en la extensión de las fronteras de su saber disciplinario de origen."

A nuestro entender, dichos objetivos carecían de instrumentos y momentos adecuados que les permitieran cumplir con ese "saber hacer" directamente orientado hacia la aplicación del conocimiento de la disciplina. De allí que nuestra primera propuesta consistió en trabajar durante el curso diseñando un artefacto que sirviera de vehículo para concretar una primera aproximación a la tesina, al que arribaríamos mediante un estudio consciente, articulando la teoría con el proceso científico a fin de arribar a una planificación y al examen de las ideas que se proponían discutir, describir, explorar o explicar, según cada caso. 
A su vez, en ese momento, las condiciones de regularidad de la materia se limitaban al cumplimiento de un solo requisito por parte del alumno: la asistencia a clases, dos días de la semana durante un total de 3 horas reloj. Por lo tanto, el artefacto en el cual estábamos pensando, y los procedimientos para desarrollarlo, también nos permitiría elaborar un ajuste en esta situación que a nuestro juicio resultaba insuficiente para definir una tesina.

Esta fue la situación normativa dentro del periodo signado por los años 2003 al 2006, ambos inclusive.

Seguidamente, en el año 2007, comienzan a proponerse y concretarse los primeros cambios en la norma vigente como consecuencia de que, adicionalmente, comenzábamos a tomar conocimiento de una cantidad significativa de alumnos que adeudaban la presentación de sus trabajos finales y que, en dos años posteriores al curso, perdían su condición de regularidad en la materia. En síntesis, de lo descripto anteriormente, se derivaron las siguientes consecuencias:

- Alumnos que debían recursar la materia.

- Mayor número de inscriptos por año.

- Saturación de los cargos de directores de tesis.

- Pérdida de la motivación del alumno para concretar los estudios de la tesina y, en la institución, cuestionamientos hacia las condiciones de realización del trabajo final.

La situación general ocasionaba la formación de un círculo vicioso ya que, si se analizan las diferentes variables mencionadas, todas ellas manifiestan conexiones entre sí.

\section{LAS PROPUESTAS SUPERADORAS}

Frente a esta situación y, cabe aclarar, una vez que nos hiciéramos cargo de la materia mediante el correspondiente concurso docente, propusimos:

a) La reformulación de la reglamentación vigente, la que hoy se traduce en la Resolución CD No 8316.

b) La modificación de las estrategias pedagógicas y didácticas de enseñanza-aprendizaje considerando las utilizadas en el período anterior (2003-2006).

Para llegar a la propuesta de hoy, año a año, todos los docentes revisamos la planificación de los contenidos y actividades, teniendo en cuenta la experiencia del año anterior; siempre buscando brindar nuevas herramientas que acerquen al alumno al proceso de investigación científica y faciliten su desarrollo hasta la culminación de la tesina.

Asimismo, con el fin de mejorar el proceso, se llegó a la redacción de una Guía de trabajos prácticos que contiene actividades que acompañan al alumno en la construcción de su plan de tesina y en la auto-evaluación de cada paso que éste realiza dentro del mismo.

Es importante aclarar y como dato a destacar en este trabajo que la experiencia nos indica que la cantidad de alumnos por curso no puede ser superior a 60 alumnos si se persigue optimizar el proceso y sus resultados. Así lo requiere la estrategia propuesta 
con el fin de ser exitosamente presentada la tesina en el tiempo esperado por la institución. Además, a ello se suman las condiciones académicas que consideramos y evaluamos como convenientes, limitando elcursado de la materia a alumnos que no posean más de 05 materias por cursar y/o rendir, ya que cuentan con mayor tiempo disponible para dedicarse a redactar el plan, concentrarse en su tesina y en las tareas de indagación que cada una de estas actividades científicas implican.

Como otra estrategia que colabora en la construcción de los conocimientos y del plan, utilizamos prioritariamente el trabajo en grupo, el que es significativo en los aportes que recibe el tesista mediante el intercambio con sus pares, quienes pueden en esta primera etapa intervenir en la selección del tema, búsqueda de antecedentes y diseño del objeto de investigación.

Las tutorías presenciales fueron cambiando en su estrategia al paso de los años, transformándose hoy en un espacio de reflexión compartido por un número no mayor a seis alumnos por grupo, que cuentan con un tiempo para exponer los avances de su plan de tesis ante sus compañeros y los docentes-guías. Este es un espacio donde se aprovecha para discutir y analizar los temas, los antecedentes, los objetivos y problema de investigación, en fin, todos los elementos componentes del instrumento de planificación. La secuencia de estas tutorías se organiza por grupos que se componen al principio del curso en función a la cantidad de alumnos. Hasta ahora, el orden es alfabético. Las tutorías tienen tres momentos en el cuatrimestre, llamados avances, y que constituyen las instancias evaluativas del proceso. En el último momento, el alumno realiza la presentación final del plan de tesina conforme a las evaluaciones y devoluciones intermedias obtenidas, las revisiones y retroalimentación recibidas en el grupo y de parte de los tutores-guías. Aprobado el plan en la última instancia, se regulariza la materia.

No menos importante es la instancia en la que hemos decidido incorporar al proceso de aprendizaje la participación de grupos de investigación y de los ex tesistas, ya graduados, mediante visitas programadas. Sus exposiciones, referidas a sus experiencias y actividades actuales como investigadores, nos permiten activar una función motivacional muy significativa en el aprendizaje que pretendemos del alumno porque le permite reconocerse a sí mismo como parte de un sistema científico dentro de la Universidad y de su futura profesión.

Es decir, hicimos una transformación integral para apoyar los cambios en las diferentes dimensiones.

Claro está, dichos cambios se produjeron en forma sucesiva y sistemática agrupados en al menos tres grandes etapas: primera, de reformulación de los objetivos de la materia y de los requisitos para acceder y regularizar la misma; segunda, reestructuración de las estrategias e instrumentos pedagógicos utilizados por la cátedra para el desarrollo de contenidos y respecto a la formulación del plan de tesina; tercera, y quizás más profunda, ajustes al proceso de corrección de la tesina, a las condiciones para ser director de tesina, la implementación de un sistema de recuperación para alumnos rezagados o que hayan perdido la regularidad en la materia. 


\section{RESULTADOS DE CADA PROPUESTA}

- Primera etapa (2007-2008)

o El Consejo Directivo aprueba bajo número de Resolución 8316 el nuevo programa de la materia, el cual involucra la reformulación de los objetivos de aprendizaje como sigue:

- "Lograr en el alumno el conocimiento básico indispensable para la investigación en el campo económico, de la empresa, del sector público y de entes sin fines de lucro.

- Aplicar los métodos correlativos al problema planteado.

- Poder seleccionar apropiadamente el material.

- Aprender a redactar un trabajo científico en el cual la discusión del tema se sigue del marco teórico y la hipótesis planteada."

o Al mismo tiempo, propusimos que el alumno que se inscriba para cursar la materia deba cumplir, como mínimo, con las siguientes condiciones:

- "Se encuentre cursando el último año de la Carrera en forma regular.

- No adeude ninguna materia teórica de los años anteriores.

- Tenga regularizadas todas las materias teóricas-prácticas del año anterior".

Mediante la implementación de estas condiciones comenzamos a lograr algunas soluciones: disminuyó el número de inscriptos al curso por año y, más importante aún, mejorar las condiciones académicas del alumno en "situación de tesis" ya que, al poseer un número menor de materias pendientes dispone de un número mayor de horas para dedicarse a preparar, durante el curso, su plan de tesina y sus exposiciones para las tutorías y, al mismo tiempo o al culminar, preparar su trabajo final de tesina.

o Incorporamos como condición de regularidad aprobar las diferentes etapas del diseño del Plan de Tesina manteniendo, en forma concomitante, la obligación de cumplir con la asistencia mínima del $75 \%$ al total de clases.

Respecto de esta primera etapa, que caracterizamos como "la transformación normativa", los resultados se comenzaron a evidenciar a partir del año 2009. Para citar un ejemplo, cabe tomar al total de alumnos que cursaron la materia durante en el año 2006, alcanzaba a 39 alumnos. De éstos, dentro del periodo de regularidad, solamente presentaron su tesina en total 04 alumnos. Y de la misma edición del curso, solamente se han graduado 18 alumnos. En cambio, en el año 2007, regularizaron la materia 52 alumnos, sobre un total de 54 inscriptos; y presentaron su tesina dentro del periodo de regularidad de la materia un total de 16 alumnos y otros 20 lo hicieron dentro del siguiente año. Es decir, un total de 36 alumnos, que representan el 69,2\% del total, llegaron al término con su tesina. Con ello podemos evidenciar que las condiciones de regularidad no afectaron los resultados de promoción del curso aunque, sin embargo, se estaban mejorando significativamente las condiciones para llegar a concretar el trabajo final.

- Segunda etapa (2009-2010)

o En este periodo propusimos profundizar aún más este cambio abordando, por ejemplo, la dimensión de los directores de tesinas y habilitando para esta función a los Auxiliares Docentes. Finalmente, la Resolución CD 9432 establece las siguientes categorías para ser Director: 
- "Profesor Titular por Concurso,

- Profesor Adjunto por Concurso, o

- Auxiliar Docente por Concurso, con Título de Especialización, Maestría o Doctorado fehacientemente acreditado.

- Se podrán observar casos especiales en función a los méritos o antecedentes equivalentes que reúna el Profesor interesado en ser Director. En estos casos los pedidos se harán por nota, dirigida al Decano, adjuntando los antecedentes que fundamenten el pedido de excepción, para que dicte Resolución si correspondiera".

A su vez, esta última opción nos resulta muy significativa ya que abre la posibilidad de insertar a nuevas personas para ocupar el rol de director, con diferentes perfiles y orígenes profesionales, ofreciendo un espectro más amplio respecto a la oferta anterior, circunscripta a la propia Casa de estudios o al plantel docente de la propia universidad. Como dato, a julio del 2011, ya se han aprobado mediante resolución del Consejo Directivo al menos 05 casos de directores de tesinas en tales condiciones, constituyendo a nuestro criterio una valiosa capitalización en materia de antecedentes para futuros tesistas.

o Otra de las dimensiones abordadas fue en torno al trabajo de la motivación de los alumnos. Conocíamos la existencia de alumnos que habían regularizado la materia y debían terminar su trabajo final en tiempo y forma pero no encontraban la conexión motivacional para hacerlo, o bien y más lejos aún, debían realizar la recuperación de su regularidad en la materia. Es así que elaboramos una propuesta que denominamos "Seminario de Recuperación de Tesistas" cuyo objetivo fundamental, como los expresamos al presentar la propuesta aprobada por Resolución CD 9156, fue "recuperar a los tesistas más rezagados en la presentación de sus trabajos finales", e incluimos también a quiénes habiendo culminado el curso en el tiempo regular de dictado no habían alcanzado a regularizar la materia con un Plan de Tesina aprobado. Esto último otorga una ventaja en el tiempo para no esperar un nuevo periodo de dictado regular, ya que el Seminario de Recuperación se realiza cada año en contracuatrimestre al dictado de la materia.

o Finalmente, modificamos el proceso de corrección de las tesinas mediante un procedimiento especialmente diseñado con el objetivo de evitar los denominados "cuellos de botella" provocados por la excesiva concentración de las tareas que implicaban la revisión metodológica de los trabajos. Es así que propusimos incluir en los cronogramas de trabajo, de los planes de tesina, un mayor intercambio y compromiso recíproco entre el director y su dirigido y que sea el director de la tesina el primer evaluador del trabajo bajo cuyo aval el alumno queda en condiciones para presentar la versión original de su trabajo para la posterior corrección metodológica. Con ello logramos que ambas partes asumieran mayores compromisos antes de la presentación del trabajo, acelerando la efectividad de nuestra tarea que, ahora con el cambio realizado, se restringió exclusivamente a revisar cinco partes de la tesina: resumen, introducción, metodología, conclusiones y bibliografía, incluidas las citas y las referencias de todo el trabajo; en oposición, antes debíamos revisar íntegramente el trabajo. El siguiente cuadro muestra el efecto marcado en el número de tesinas que se defendieron entre 2010 y lo que llevamos al mes de Julio del año 2011: 


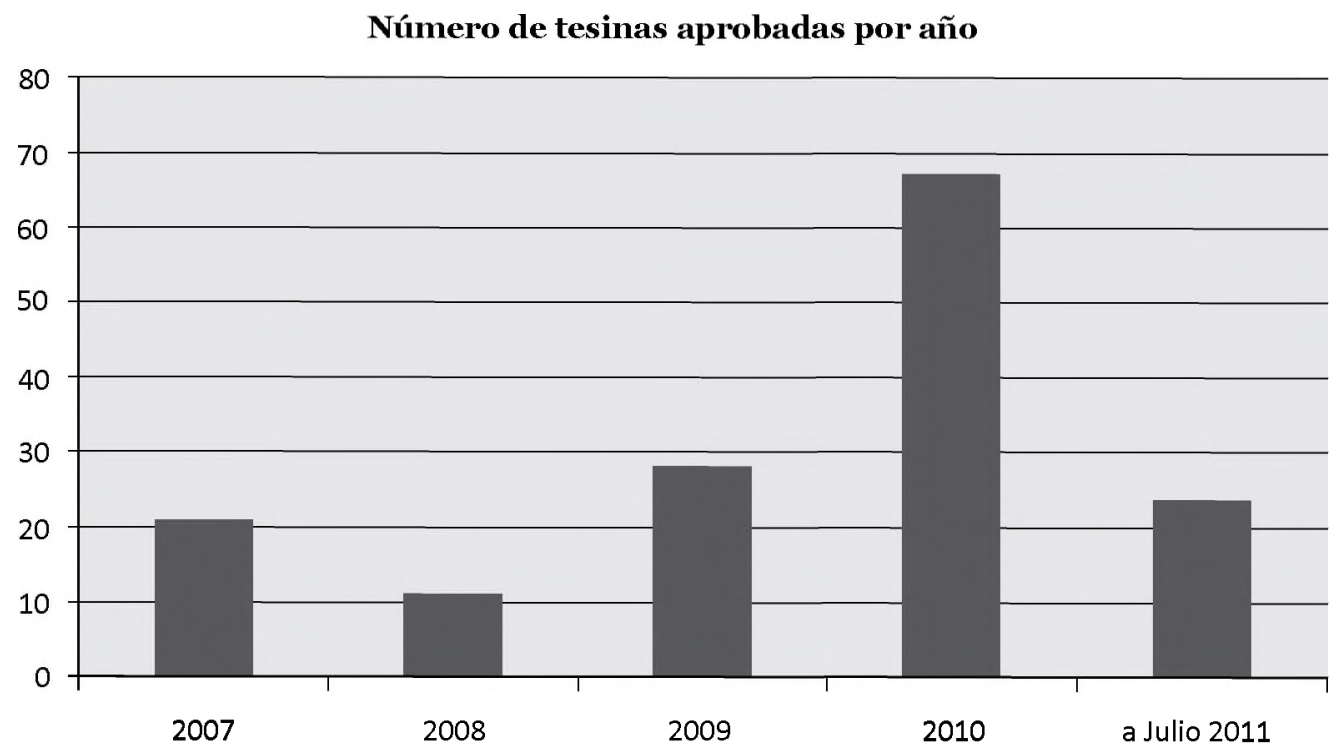

Fuente: elaboración propia en base a datos de la Cátedra.

o Otras de las dimensiones más interesantes para destacar respecto a la producción científica se relaciona con las temáticas seleccionadas para abordar por los alumnos. La selección de los temas surge a partir de un proceso de reflexión e intercambios intensivos que se producen durante la primera etapa del curso y refleja, por un lado, un compromiso singular con el análisis y solución de los problemas de índole local y, por el otro, la impronta de originalidad de un verdadero trabajo de tesis cuyos niveles de producción del conocimiento en muchos casos se aproxima a una tesis de posgrado. Como ejemplo, se anexa información sobre los temas y los respectivos autores de las tesinas defendidas entre los años 2010 y 2011.

\section{CONCLUSIONES}

Los cambios que hemos compartido en esta publicación forman parte de un proceso que continúa de manera permanente como parte de la constante reflexión sobre nuestra práctica docente y sus resultados, y no solamente significó una modificación en el enfoque del trabajo que debíamos realizar en la materia sino que también significó un nuevo compromiso con el alumno en el sentido de acompañar su proceso de investigación y ayudarlo a obtener su producto final: un trabajo científico cuyos stándares de calidad fueran meritorios de un esfuerzo de formación comprometido y de excelencia en los marcos de una Academia como la UNNE.

Participamos de mantener la tesina final dentro de los planes de estudio de las Licenciaturas ya que existen diferentes estrategias que, como se ha demostrado, pueden ser implementadas a fin de lograr una mejor producción científica, haciendo del Licenciado en Administración y Eco- 
nomía, egresados de la Facultad de Ciencias Económicas de la UNNE, un profesional preparado para los desafíos y necesidades que tanto el mercado como la sociedad plantea en el siglo XXI .

En miras al futuro de nuestras investigaciones de cátedra continuamos analizando los resultados que se han registrado y derivando de ellos las nuevas estrategias que los problemas que surgen nos van imponiendo, tales como encontrar nuevos nichos en el campo del conocimiento de ambas disciplinas, Administración y Economía, y líneas de análisis para futuras investigaciones.

\section{ANEXO: TEMAS Y AUTORES DE TESINAS} DEFENDIDAS EN EL PERIODO 2010 - 2011

\begin{tabular}{|c|c|c|c|}
\hline № & NOMBRE Y APELLIDO & TEMA & TUTOR \\
\hline 1 & $\begin{array}{l}\text { SZYMULA } \\
\text { Carolina María Isabel }\end{array}$ & $\begin{array}{l}\text { Pérdida de Competitividad del Puerto de } \\
\text { Barranqueras en el Período } 1980-2008 .\end{array}$ & Cr. Gerardo Santos Oliveira \\
\hline 2 & $\begin{array}{c}\text { OCAMPO } \\
\text { Amalia Virginia }\end{array}$ & $\begin{array}{l}\text { Los sistemas de apoyo al emprendedorismo, } \\
\text { otorgados por organizaciones gubernamentales } \\
\text { y no gubernamentales de Resistencia. }\end{array}$ & Cr. Marcón \\
\hline 3 & $\begin{array}{c}\text { LESCANO } \\
\text { María Eugenia }\end{array}$ & $\begin{array}{c}\text { Aplicación del Marketing en las organziaciones } \\
\text { no gubernamentales de la ciudad de } \\
\text { Montecarlo y El Dorado. }\end{array}$ & Lic. Odriozola \\
\hline 4 & $\begin{array}{l}\text { VALIÑO } \\
\text { María Laura }\end{array}$ & $\begin{array}{l}\text { Los conflictos entre grupos en una } \\
\text { organización sin fines de lucro. }\end{array}$ & Cr. Marcón \\
\hline 5 & $\begin{array}{l}\text { PALMEYRO } \\
\text { Matías }\end{array}$ & $\begin{array}{l}\text { Análisis de la cadena productiva forestal } \\
\text { de la Provincia de Corrientes. }\end{array}$ & Dr. Lucas Ferrero \\
\hline 6 & $\begin{array}{c}\text { KATZ } \\
\text { Benjamín }\end{array}$ & $\begin{array}{l}\text { Análisis de la evolución del sector forestal } \\
\text { de Corrientes y sus perspectivas. }\end{array}$ & Cr. Marcón \\
\hline 7 & CENA María Laura & Sector arrocero correntino. & Mgter. William García \\
\hline 8 & $\begin{array}{l}\text { HIDALGO Gabriela } \\
\text { Patricia }\end{array}$ & $\begin{array}{l}\text { Cultura Organizacional. Aplicabilidade } \\
\text { incidenciaen una empresa charatense. }\end{array}$ & Mgter. William García \\
\hline 9 & $\begin{array}{l}\text { SEQUEIRA CHILOTEGUI } \\
\text { José }\end{array}$ & $\begin{array}{l}\text { Tablero de mando integral en empresas } \\
\text { de transporte de la Ciudad de Corrientes. }\end{array}$ & Dr. Dellamea \\
\hline 10 & $\begin{array}{l}\text { ZARRABASKY } \\
\text { Virginia }\end{array}$ & $\begin{array}{c}\text { El papel de la cultura organizacional y comu- } \\
\text { nicación interna en la Asociación Mutual de } \\
\text { profesionales de salud bucal. }\end{array}$ & Cr. Marcón \\
\hline 11 & $\begin{array}{l}\text { BUTTICE } \\
\text { Ana Clara }\end{array}$ & $\begin{array}{l}\text { El microcrédito como herramienta de abordaje } \\
\text { territorial para las organizaciones de Gran Resis- } \\
\text { tencia, en su rol de promotoras del desarrollo. }\end{array}$ & Cr. Rubén Marcón \\
\hline 12 & $\begin{array}{l}\text { MORENO CENOZ } \\
\text { María }\end{array}$ & Estructuras organizacionales. & Cr. Marcón \\
\hline
\end{tabular}




\begin{tabular}{|c|c|c|c|}
\hline № & NOMBRE Y APELLIDO & TEMA & TUTOR \\
\hline 13 & $\begin{array}{l}\text { SHEIDEGGER } \\
\text { Gabriela }\end{array}$ & $\begin{array}{c}\text { El descuento de cheques de pago diferido en el } \\
\text { mercado de capitales. Su utilización por parte } \\
\text { de pequeñas y medianas empresas } \\
\text { de la Ciudad de Formosa. }\end{array}$ & Lic. Fiol \\
\hline 14 & $\begin{array}{c}\text { DAVIÑA } \\
\text { María Mercedes }\end{array}$ & $\begin{array}{l}\text { Aspectos motivacionales que influyen en el } \\
\text { comportamiento de compra de jóvenes } \\
\text { correntinas de clase media en vestimenta }\end{array}$ & Lic. Odriozola \\
\hline 15 & $\begin{array}{c}\text { ENCINA } \\
\text { Mirtha Edith }\end{array}$ & $\begin{array}{c}\text { Responsabilidad social empresaria en las } \\
\text { empresas constructoras de Corrientes: } \\
\text { ¿realidad o ficción? }\end{array}$ & Cr. Campetella \\
\hline 16 & $\begin{array}{l}\text { PEGORARO } \\
\text { Laura }\end{array}$ & $\begin{array}{c}\text { Sistema de innovación aplicado a la industria de } \\
\text { software y servicios informáticos de } \\
\text { la Provincia del Chaco. }\end{array}$ & Lic. Nievas \\
\hline 17 & $\begin{array}{l}\text { VIDAL GARCÍA } \\
\text { Carlos }\end{array}$ & $\begin{array}{l}\text { Análisis de la productividad del personal } \\
\text { de servicio de salud de una } \\
\text { clínica de la Ciudad de Corrientes. }\end{array}$ & Lic. Odriozola \\
\hline 18 & $\begin{array}{l}\text { MICHICHE } \\
\text { Angelina }\end{array}$ & $\begin{array}{c}\text { La gestión y estructura organizativa } \\
\text { de una empresa Familiar. }\end{array}$ & Cr. Marcón \\
\hline 19 & $\begin{array}{c}\text { BALISTRERI } \\
\text { Germán Emanuel }\end{array}$ & $\begin{array}{l}\text { Cultura Organizacional. Aplicabilidade } \\
\text { incidenciaen una empresa charatense. }\end{array}$ & Cr. Marcón \\
\hline 20 & $\begin{array}{l}\text { SCHAFHEUTLE } \\
\text { Karina Verónica }\end{array}$ & $\begin{array}{c}\text { Gestión estratégica de una cooperativa } \\
\text { agroindustrial de Corrientes. }\end{array}$ & Cr. Marcón \\
\hline 21 & $\begin{array}{l}\text { WACJMAN } \\
\text { Cecilia }\end{array}$ & $\begin{array}{c}\text { Rol de los Planes sociales: Su incidencia en el } \\
\text { Desarrollo Socioeconómico, la } \\
\text { Reproducción cultural y el Estigma. }\end{array}$ & Lic. Alicia Carlino \\
\hline 22 & $\begin{array}{l}\text { CABRERA } \\
\text { María de los Ángeles }\end{array}$ & $\begin{array}{l}\text { Indicadores de Responsabilidad social } \\
\text { empresaria sobre información y publicidad } \\
\text { a consumidores en concesionaros } \\
\text { automotres de Resistencia. }\end{array}$ & Cra. Rossana N. Greco \\
\hline 23 & $\begin{array}{l}\text { RODRIGUEZ } \\
\text { Mirna Silvana }\end{array}$ & Las Organizaciones apicolas y sus estructuras. & Cr. Jorge Zárate \\
\hline 24 & $\begin{array}{c}\text { RIVERO } \\
\text { Lorena Alejandra }\end{array}$ & $\begin{array}{l}\text { El Proceso Administrativo en } \\
\text { las Cooperativas del Medio. }\end{array}$ & Dra. Kesselman \\
\hline 25 & $\begin{array}{l}\text { SOSA LEYES } \\
\text { Carolina }\end{array}$ & $\begin{array}{c}\text { El trabajo invisible. El trabajo } \\
\text { doméstico en la Ciudad de Corrientes. }\end{array}$ & Dra. Kesselman \\
\hline 26 & $\begin{array}{l}\text { GONZALEZ DADONE } \\
\text { Martín Alfredo }\end{array}$ & $\begin{array}{l}\text { Ferias Francas en la Ciudad de Corrientes. Or- } \\
\text { ganización, participación y vínculos de actores } \\
\text { sociales en su conformación (2008-2010). }\end{array}$ & Dra. Elena Alfonso \\
\hline 27 & $\begin{array}{l}\text { LOBEL } \\
\text { Marilyn Desiree }\end{array}$ & $\begin{array}{l}\text { Las cooperativas agricolas } \\
\text { y su cultura organizacional. }\end{array}$ & Dr. Oscar Campetella \\
\hline
\end{tabular}




\begin{tabular}{|c|c|c|c|}
\hline № & Nombre y Apellido & Tema & Tutor \\
\hline 28 & $\begin{array}{l}\text { VIGGIANO PONT } \\
\text { Pedro Eduardo }\end{array}$ & $\begin{array}{l}\text { Determinación de las cualidades del perfil em- } \\
\text { prendedor de los Licenciados en Administración } \\
\text { de la Facultad de Cs. Ec. De la UNNE. }\end{array}$ & Dr. César Dellamea \\
\hline 29 & $\begin{array}{l}\text { HABERMACHER } \\
\text { Daniel Federico }\end{array}$ & $\begin{array}{l}\text { Sostenibilidad de las Finanzas Públicas Provincia- } \\
\text { les: fundamentos del modelo fiscal provincial y } \\
\text { proyecciones de comportamiento 2008-2015. }\end{array}$ & Dr. Lucas Ferrero \\
\hline 30 & $\begin{array}{l}\text { ZARRABEITIA } \\
\text { César Tomás }\end{array}$ & $\begin{array}{l}\text { La Ciudad de invierno, Orígenes, Evolu- } \\
\text { ción y fracaso del Proyecto turístico e } \\
\text { inmobiliario"Mansión de invierno". }\end{array}$ & Lic. Alicia Carlino \\
\hline 31 & $\begin{array}{l}\text { MONTI } \\
\text { Evelin }\end{array}$ & $\begin{array}{l}\text { Ética y responsabilidad social empresaria como } \\
\text { eje central para el Desarrollo sustentable. }\end{array}$ & Cr. José María Labrousse \\
\hline 32 & $\begin{array}{l}\text { BRAUN BILLINGHURT } \\
\text { Lautaro }\end{array}$ & $\begin{array}{l}\text { Técnicas para motivar al personal } \\
\text { en una empresa familiar. }\end{array}$ & Dr. Oscar Campetella \\
\hline 33 & $\begin{array}{l}\text { TOGNOLA } \\
\text { Sandra Mara }\end{array}$ & $\begin{array}{c}\text { Las repercusiones socioeconómicas y ambientales } \\
\text { del Proyecto de Instalación de un Parque Indus- } \\
\text { trial en Puerto Vilelas, según la perspectiva } \\
\text { de los actores sociales involucrados. }\end{array}$ & Cr. Rubén Marcón \\
\hline 34 & $\begin{array}{l}\text { MITRE } \\
\text { Melina }\end{array}$ & $\begin{array}{c}\text { Perspectiva del Empresario de Resistencia, } \\
\text { socialmetne responsable } \\
\text { con su comunidad. }\end{array}$ & Prof. Marta Trógolo \\
\hline 35 & $\begin{array}{c}\text { IBARRA } \\
\text { María de los Milagros }\end{array}$ & Gestión de la seguridad y la salud. & Ing. Lilia Marcela Báez \\
\hline 36 & $\begin{array}{l}\text { FONTAN } \\
\text { Carolina }\end{array}$ & Inversión e incertidumbre en Argentina. & Dr. Lucas Ferrero \\
\hline 37 & $\begin{array}{l}\text { MIERES ESTIGARRIBIA } \\
\text { Mauro Gastón }\end{array}$ & $\begin{array}{l}\text { La competitividad internacional del sector agrico- } \\
\text { la argentino para el periodo 1990-2009. }\end{array}$ & Dra. Elena Alfonso \\
\hline 38 & ESTOUP Jorge & $\begin{array}{l}\text { Comparación de la Productividad del factor } \\
\text { trabajo en empresas familiares y no familiares. }\end{array}$ & Cr. Rubén Marcón \\
\hline 39 & $\begin{array}{l}\text { PUGLIESE } \\
\text { Lisandro }\end{array}$ & $\begin{array}{l}\text { Neuromarketing en la comercialización de } \\
\text { electrodomésticos en el NEA Caso Megatone. }\end{array}$ & Dr. César Dellamea \\
\hline 40 & $\begin{array}{l}\text { POLETTI } \\
\text { Florencia }\end{array}$ & $\begin{array}{c}\text { Efectos del gasto público vinculado al programa } \\
\text { ganadero del noroeste chaqueño en el creci- } \\
\text { miento del valor agregado del sector caprino } \\
(1999-2008)\end{array}$ & Lic. Daniela Torrente \\
\hline 41 & $\begin{array}{l}\text { BAROZZI } \\
\text { María de los Ángeles }\end{array}$ & $\begin{array}{l}\text { Posibilidades de desarrollo de la industria de la } \\
\text { confección de jeans en la Provincia del Chaco. }\end{array}$ & Dra. Elena Alfonso \\
\hline 42 & $\begin{array}{l}\text { ETCHELOUZ } \\
\text { Franco Martín }\end{array}$ & $\begin{array}{l}\text { Aspectos estratégicos y organizativos en } \\
\text { servicios de estética corporal en Resistencia. }\end{array}$ & Mgter. Guillermo Odriozola \\
\hline 43 & $\begin{array}{l}\text { BORFITZ } \\
\text { María Silvina }\end{array}$ & $\begin{array}{c}\text { Caracteristicas de la Cultura Organizacional de } \\
\text { una empresa de Familia de Resistencia. }\end{array}$ & Dr. Oscar Campetella \\
\hline
\end{tabular}




\begin{tabular}{|c|c|c|c|}
\hline № & NOMBRE Y APELLIDO & TEMA & TUTOR \\
\hline 44 & $\begin{array}{c}\text { ACEVEDO } \\
\text { Eduardo Esteban }\end{array}$ & $\begin{array}{l}\text { Evaluación de Proyectos de Inversión. La asig- } \\
\text { nación de recursos públicos y su impacto social. }\end{array}$ & Cr. Gerardo Santos Oliveira \\
\hline 45 & $\begin{array}{l}\text { DAL' MOLIN } \\
\text { María Laura }\end{array}$ & $\begin{array}{l}\text { Cadena de Valor de la Mandarina como Fruta } \\
\text { Fresca desde la localidad de Mocoretá, provin- } \\
\text { cia de Corrientes, hasta Rótterdam, Holanda. }\end{array}$ & Dr. César Dellamea \\
\hline 46 & $\begin{array}{l}\text { LESCANO ORAISON } \\
\text { María Cecilia }\end{array}$ & $\begin{array}{l}\text { Las Marias: Caso paradigmático de relación co- } \\
\text { munitaria y responsabilidad social empresaria, } \\
\text { en la localidad de Gobernador Valentin Virasoro } \\
\text { y zona de influencia, Provincia de Corrientes. }\end{array}$ & Dr. César Dellamea \\
\hline 47 & $\begin{array}{l}\text { LHEMANN } \\
\text { Flavio Ernesto }\end{array}$ & $\begin{array}{c}\text { Preferensicas organilépticas de la carne de } \\
\text { cerdo en opiniones de consumidores de la } \\
\text { Ciudad de Resistencia. }\end{array}$ & Mgter. Guillermo Odriozola \\
\hline 48 & $\begin{array}{c}\text { HESS } \\
\text { Ricardo Elfio }\end{array}$ & $\begin{array}{l}\text { Aplicación de la matriz grupo consultor de } \\
\text { Boston a Pymes de la Ciudad de Resistencia. }\end{array}$ & Lic. Millán \\
\hline 49 & $\begin{array}{l}\text { ORTIZ ALMIRON } \\
\text { Nélida Yanina }\end{array}$ & $\begin{array}{l}\text { Fideicomisos financieros en } \\
\text { las Pymes Industriales. }\end{array}$ & Cra. Margarita Carbajal \\
\hline 50 & $\begin{array}{l}\text { DOMINGORENA } \\
\text { Leandro Horacio }\end{array}$ & $\begin{array}{c}\text { Identificación y Análisis de obstaculizadores } \\
\text { en la Gesitón de los residuos sólidos } \\
\text { urbanos en Resistencia. }\end{array}$ & Dr. César Dellamea \\
\hline 51 & $\begin{array}{l}\text { FRENCIA } \\
\text { Paola }\end{array}$ & Sobre la dinámica del empleo y las empresas. & Lic. Lucas Ferrero \\
\hline 52 & $\begin{array}{c}\text { KUCHARUK } \\
\text { Diego Sebastián }\end{array}$ & $\begin{array}{c}\text { Análisis de la Responsabilidad Social Empresa- } \\
\text { ria en el área de capacitación y su cultura } \\
\text { de una Pyme industrial. }\end{array}$ & Dr. Oscar Campetella \\
\hline 53 & $\begin{array}{l}\text { GALLARDO } \\
\text { José Alberto }\end{array}$ & Administración, Eficiencia y Lechería. & Prof. Esp. Gricela Rohde \\
\hline 54 & $\begin{array}{l}\text { SÁNCHEZ DANSEY } \\
\text { María Adriana }\end{array}$ & $\begin{array}{c}\text { Cadena de Valor en Empresa } \\
\text { Ganadera EI Olvido. }\end{array}$ & Mgter. Williams García \\
\hline 55 & $\begin{array}{c}\text { GIMENEZ } \\
\text { Federico Rubén }\end{array}$ & $\begin{array}{c}\text { Cultura Organizacional de una cooperativa } \\
\text { de la Provincia de Corrientes. Influencia de los } \\
\text { valores y principios cooperativos. }\end{array}$ & Dra. Silvia Kesselman \\
\hline 56 & $\begin{array}{l}\text { SUAREZ } \\
\text { Gabriel Hernán }\end{array}$ & $\begin{array}{l}\text { El gobierno local de Yapeyú -el Municipio- sus } \\
\text { fortalezas con las nuevas facultades otorgadas } \\
\text { con la enmienda constitucional de } 2007 .\end{array}$ & Cr. Zárate \\
\hline 57 & $\begin{array}{l}\text { PEGORARO } \\
\text { Mónica }\end{array}$ & $\begin{array}{c}\text { Percepción del consumidor de carne de cerdo. } \\
\text { Mercado Resistencia. }\end{array}$ & Mgter. Guillermo Odriozola \\
\hline 58 & $\begin{array}{l}\text { DELLAMEA LIVA } \\
\text { Pedro Javier }\end{array}$ & $\begin{array}{l}\text { Construcción de un marco conceptual integrador } \\
\text { mediante el uso de teorías de gestión del cono- } \\
\text { cimiento para el diagnóstico y adecuación de las } \\
\text { tecnologías de información, los recursos humanos } \\
\text { y la estructura organizacional en los procesos. }\end{array}$ & Ing. Gustavo Devicenzi \\
\hline
\end{tabular}




\begin{tabular}{|c|c|c|c|}
\hline № & NOMBRE Y APELLIDO & TEMA & TUTOR \\
\hline 59 & $\begin{array}{l}\text { INGARAMO } \\
\text { Nicolás }\end{array}$ & $\begin{array}{l}\text { Estrategias empresariales para la expansión } \\
\text { geográfica en el nordeste argentino. }\end{array}$ & Dra. Mónica Canteros \\
\hline 60 & $\begin{array}{l}\text { GOITIA } \\
\text { Nur llene }\end{array}$ & $\begin{array}{l}\text { Determinación del grado de aplicación de Respon- } \\
\text { sabilidad Social Empresaria en la dimensión Públi- } \\
\text { co interno de las cooperativas de Barranqueras. }\end{array}$ & Dra. Kesselman \\
\hline 61 & $\begin{array}{l}\text { PACHECO } \\
\text { Héctor Leandro }\end{array}$ & $\begin{array}{l}\text { Factores Gerenciales claves para el crecimiento } \\
\text { sustentable de las Pymes Chaqueñas. }\end{array}$ & Cr. Marcón \\
\hline 62 & $\begin{array}{l}\text { ALARCÓN } \\
\text { Claudia }\end{array}$ & $\begin{array}{l}\text { Comportamiento de compra de clientes de } \\
\text { agencias de viajes de la ciudad de Resistencia. }\end{array}$ & Lic. Silvina Marina Blanqué \\
\hline 63 & $\begin{array}{l}\text { MACUGLIA } \\
\text { Damián }\end{array}$ & $\begin{array}{l}\text { El rol de las expectativas individuales con respecto } \\
\text { a la evolución del PIB y su efecto sobre la deman- } \\
\text { da de Inversión en Argentina. 2002-2010. }\end{array}$ & Dra. Elena Alfonso \\
\hline 64 & $\begin{array}{l}\text { PAREDES } \\
\text { Nadia }\end{array}$ & $\begin{array}{c}\text { Efectos de estrategias alternativas de gestión } \\
\text { de riesgos en los ingresos de explotaciones } \\
\text { agrícolas de la Provincia del Chaco. }\end{array}$ & Dr. Lucas Ferrero \\
\hline 65 & $\begin{array}{c}\text { GUZMÁN } \\
\text { Fernando Emanuel }\end{array}$ & $\begin{array}{c}\text { Dinámica de la pobreza en el Gran Resistencia } \\
\text { durante el periodo 2003-2006 y sus principales } \\
\text { condiconantes socioeconómicos. }\end{array}$ & Dra. Alfonso \\
\hline 66 & $\begin{array}{l}\text { SAN JOSÉ } \\
\text { Mario Alejandro }\end{array}$ & $\begin{array}{c}\text { Medidas de pobreza, una aproximación } \\
\text { a las disparidades regionales. }\end{array}$ & Dr. Lucas Ferrero \\
\hline 67 & $\begin{array}{c}\text { CATAY } \\
\text { Adriana Mabel }\end{array}$ & $\begin{array}{l}\text { Un compromiso con la calidad: Caso de una } \\
\text { Cooperativa Citricola "Colonia San Francisco" } \\
\text { Monte Caseros - Provincia de Corrientes. }\end{array}$ & Dra. Alfonso \\
\hline 68 & $\begin{array}{l}\text { MASTANDREA } \\
\text { Natalia }\end{array}$ & $\begin{array}{c}\text { El cuadro de Mando Integral como herramienta } \\
\text { de Gestión estratégica desde la perspecitva de } \\
\text { Aprendizaje y Crecimiento. }\end{array}$ & Cr. Celestino Fantin \\
\hline 69 & $\begin{array}{l}\text { PUCCIARELO } \\
\text { Josefina }\end{array}$ & $\begin{array}{l}\text { El desarrollo sustentable de las empresas } \\
\text { arroceras en la Provincia de Corrientes }\end{array}$ & Cr. Rubén Abel Marcón \\
\hline 70 & $\begin{array}{l}\text { PETCOFF } \\
\text { Pablo Emilio }\end{array}$ & $\begin{array}{l}\text { Aplicación de la Teoría de las Restricciones } \\
\text { en el sector foresto industrial de armado y } \\
\text { manufactura de pallets del departamento } \\
\text { Comandante Fernández (Chaco) }\end{array}$ & Ing. Germán Camprubí \\
\hline 71 & $\begin{array}{l}\text { CAMORS } \\
\text { Guido Andrés }\end{array}$ & $\begin{array}{l}\text { Cultura Organizacional de } \\
\text { El Fogón de los Arrieros. }\end{array}$ & Dr. Oscar Campetella \\
\hline 72 & $\begin{array}{c}\text { AGUIRRE } \\
\text { Adriana Esther }\end{array}$ & $\begin{array}{c}\text { Consecuencias que a nivel estratégico produce } \\
\text { la aplicación de Responsabilidad Social } \\
\text { Empresaria en empresas de seguro que } \\
\text { operan en la Provincia del Chaco. }\end{array}$ & $\begin{array}{l}\text { Dra. Maria Laura } \\
\text { Estigarribia Bieber }\end{array}$ \\
\hline 73 & $\begin{array}{l}\text { LORENTE } \\
\text { Analía Asunción }\end{array}$ & $\begin{array}{c}\text { Tenconologías de la información y la comuni- } \\
\text { cación como herramientas coadyuvantes en la } \\
\text { consolidación de la Gesitón Pública } \\
\text { de la Ciudad de Corrientes. }\end{array}$ & Cr. Jorge Zárate \\
\hline
\end{tabular}




\begin{tabular}{|c|c|c|c|}
\hline № & Nombre y Apellido & Tema & Tutor \\
\hline 74 & $\begin{array}{l}\text { MARTÍNEZ LEONE } \\
\text { Mariana Alejandra }\end{array}$ & $\begin{array}{l}\text { Estudio Comparativo de la cultura organizacio- } \\
\text { nal de dos empresas de un mismo sector. }\end{array}$ & Dra. Silvia Kesselman \\
\hline 75 & $\begin{array}{c}\text { KBAL } \\
\text { Virginia }\end{array}$ & $\begin{array}{l}\text { Evaluación de desempeño: la empresa } \\
\text { Cervecería y Maltería Quilmes reinvindica la } \\
\text { utilidad de la evaluación de desempeño. }\end{array}$ & Mgter. María Azul Forclaz \\
\hline 76 & $\begin{array}{l}\text { ORTIZ DELGADO } \\
\text { Gimena Soledad }\end{array}$ & $\begin{array}{l}\text { La calidad como factor que provoca } \\
\text { lealtad en los huéspedes hoteleros } \\
\text { de la Ciudad de Resistencia. }\end{array}$ & Ing. Hugo Maldonado \\
\hline 77 & $\begin{array}{l}\text { ORTIZ } \\
\text { Erica }\end{array}$ & $\begin{array}{c}\text { Rol del Licenciado en administración } \\
\text { en Entidades Financieras. }\end{array}$ & Lic. Jorge Fiol \\
\hline 78 & $\begin{array}{l}\text { GONZÁLEZ } \\
\text { Gisela }\end{array}$ & $\begin{array}{l}\text { Creación de Valor desde la Administración: } \\
\text { estudio de caso de una entidad financiera. }\end{array}$ & Esp. Jorge Fiol \\
\hline 79 & $\begin{array}{l}\text { SORIA OJEDA } \\
\text { Alcira }\end{array}$ & $\begin{array}{l}\text { Turismo y Competividad en la } \\
\text { Provincia del Chaco. }\end{array}$ & Lic. Marcelo Nievas \\
\hline 80 & $\begin{array}{l}\text { FERNÁNDEZ } \\
\text { Lliana Inés }\end{array}$ & $\begin{array}{c}\text { Evaluación de la ventaja competitiva de la } \\
\text { Atención al Cliente en una distribuidora de } \\
\text { productos de la Ciudad de Corrientes. }\end{array}$ & Dra. Hilda Zárate \\
\hline 81 & $\begin{array}{l}\text { ALEGRE } \\
\text { Matías }\end{array}$ & $\begin{array}{l}\text { Poder de Compra en las Pymes } \\
\text { Industriales del Gran Resistencia. }\end{array}$ & Cr. Rubén Marcón \\
\hline 82 & $\begin{array}{l}\text { RIVERO TOST } \\
\text { Daiana Ximena }\end{array}$ & $\begin{array}{c}\text { Pymes de familia y sus características en torno a } \\
\text { la denominada sucesión de poder. }\end{array}$ & Cr. Jorge Zárate \\
\hline 83 & $\begin{array}{l}\text { ARRALDE } \\
\text { Lucía Cristina }\end{array}$ & $\begin{array}{l}\text { Reclutamiento y selección del Personal en las } \\
\text { Cooperativas de la Ciudad de Corrientes. }\end{array}$ & Dra. Silvia Kesselman \\
\hline 84 & $\begin{array}{l}\text { RECALDE } \\
\text { Germán César }\end{array}$ & $\begin{array}{l}\text { Burbujas financieras: El fenómeno que no } \\
\text { pasa de moda en la Economía Mundial. }\end{array}$ & Lic. Omar Quirelli \\
\hline 85 & $\begin{array}{l}\text { MACIEL PERRONI } \\
\text { Gonzalo Eduardo }\end{array}$ & $\begin{array}{l}\text { Efecto de la normativa Nacional y Provincial } \\
\text { de Promoción sobre el Circuito Productivo } \\
\text { Forestal de la Provincia de Corrientes. }\end{array}$ & Dra. Miriam Curletti \\
\hline 86 & $\begin{array}{l}\text { SAMPOR } \\
\text { Pamela }\end{array}$ & $\begin{array}{l}\text { Unidireccionalidad de los recursos humanos } \\
\text { hacia los nuevos objetivos estratégicos de una } \\
\text { organización del medio local. }\end{array}$ & Dr. Dellamea César \\
\hline
\end{tabular}




\section{CURRICULUM VITAE MÓNICA INÉS CESANA BERNASCONI}

Contadora Pública (1996- UNNE). Especialista en Tributación (2000-UNNE). Magíster en Epistemología y Metodología de la Investigación Científica (2003-UNNE). Profesor Adjunto por concurso de Metodología de la Investigación, Seminario con Tesina Final. Licenciatura en Economía y Licenciatura en Administración. (FCE-UNNE). Jefe de Trabajos Prácticos por concurso de Impuestos I. Carrera de Contador (FCE-UNNE). Docente Investigador y de Posgrado en Maestrías, Especializaciones y Cursos de Metodología de la Investigación Científica. Autora y coautora de Publicaciones Científicas con y sin referato. Miembro de Comisiones Científicas. Doctorado en Ciencias Cognitivas (UNNE), Tesis en curso.

mcesana@eco.unne.edu.ar

\section{MÓNICA ALEJANDRA CANTEROS}

Abogada.(1993 UNNE) Especialista en Docencia Universitaria.(2005-UNNE) Docente Metodología de la Investigación, Seminario con Tesina Final. Licenciatura en Economía y Licenciatura en Administración. (FCE - UNNE). Docente con Mayor dedicación de la Asignatura Instituciones del Derecho Privado I. Coordinadora del Modulo de Derecho Introducción a las Ciencias Económicas. Investigadora Categorizada de Ciencia y Técnica de la UNNE. Integrante de Proyectos de Investigación acreditados desde el año 1998. Docente de Posgrados en Derecho y Metodología de Investigación. Autora de artículos científicos, capítulos de Libros nacionales y del exterior. Maestría en Docencia Universitaria. Tesis en curso .Alumna del Doctorado en Ciencias Cognitivas - UNNE.

mcanteros@eco.unne.edu.ar 